\title{
Plasmacytoid dendritic cells and type I interferons in flares of systemic lupus erythematosus triggered by COVID-19
}

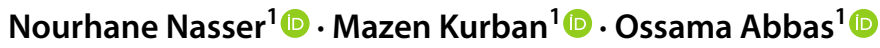 \\ Received: 19 February 2021 / Accepted: 25 February 2021 / Published online: 5 March 2021 \\ (c) The Author(s), under exclusive licence to Springer-Verlag GmbH Germany, part of Springer Nature 2021
}

Keywords SARS-Cov-2 $\cdot$ SLE $\cdot$ Plasmacytoid dendritic cell $\cdot$ Interferon

\section{Dear Editor,}

We recently read with interest the article entitled "Can the SARS-CoV-2 infection trigger systemic lupus erythematosus? A case-based review" by Gracia-Ramos et al. [1]. The authors reviewed emerging cases of SARS-CoV-2 infection either flaring-up previously diagnosed-SLE or triggering de novo SLE, and briefly pondered on the underlying mechanistic pathways leading to this association [1]. Here, we would like to highlight the plasmacytoid dendritic cell (pDC) and its major product, type I interferons (IFNs), as a possible link that may explain SLE development or flare-up during the course of SARS-Cov-2 infection.

Being unique DCs with plasma cell morphology, pDCs express CD4, HLA-DR, CD123, blood-derived dendritic cell antigen-2 (BDCA-2) and Toll-like receptor (TLR)7 and TLR9 in endosomal compartments [2-4]. They are crucial mediators of innate immunity as they sense nucleic acids via TLR7 and TLR9. Upon triggering of TLR7/9, pDCs get activated leading to massive production of type I IFNs that is mainly involved in immunity against viruses. Actually, pDCs are the most potent producers of type I IFNs, which, in turn, are critical cytokines in the control of viral replication by inducing the expression of multiple genes that lead mainly to an antiviral state. pDCs also contribute to the adaptive immunity and to the regulation of myeloid DC, T-, B- and natural killer (NK) cell function through the type I IFNs and proinflammatory cytokines that it produces such as interleukin (IL)- 6 and tumor necrosis factor (TNF)- $\alpha$. In effect, the coronaviruses including the SARS-Cov-2 are not

Ossama Abbas

ossamaabbas2003@yahoo.com

1 Department of Dermatology, American University of Beirut Medical Center, P.O. Box 11-0236, Riad El Solh 1107 2020, Lebanon exempt from the antiviral activity of the pDCs [5]. Recent evidence has demonstrated that SARS-CoV-2, like other coronaviruses, is an efficient pDC stimulator leading to strong induction of type I IFNs. In addition to helping clear up the viral infection, pDCs and the type I IFNs have been shown to contribute to cutaneous manifestations of the SARS-CoV-2 infection such as COVID-19 associated chilblains [6].

The critical role pDCs and type I IFNs play in LE pathogenesis is well-established $[2,7]$. In SLE, pDCs have been identified as the main type I IFN producers and immune complexes made up of nuclear antigens and autoantibodies are able to activate pDCs in a TLR-dependent manner Circulating pDCs are diminished in SLE patients, as they tend to migrate and infiltrate target tissues [2]. In an experimental mouse model, it has been demonstrated that early transient pDC ablation before disease initiation ameliorated LE. In addition, studies have consistently demonstrated large numbers of IFN-producing pDCs in skin LE lesions [2, 4]. As for type I IFNs, strong up-regulation of IFN-inducible genes occurs in peripheral blood of SLE patients with active disease $[2,7]$. In experimental mouse SLE models, type I IFNs have been shown to control the severity and onset of autoimmune features and introducing a null IFN receptor gene mutation clearly diminishes SLE-like disease [2]. Similarly, reports of COVID-19 inducing other CTDs in which pDCs and type I interferons, such as myositis/dermatomyositis, are also emerging $[2,8]$.

Gracia-Ramos et al. have considered the presence of a hypothetical overlapping immunologic link that may explain the association of SARS-CoV-2 infection and SLE. Based on the above presented evidence, we believe that the type I IFN-mediated protective immune response pDCs provide against viruses such as during the SARS-CoV-2 infection, may elicit IFN-driven autoimmune diseases such as SLE in genetically predisposed individuals. 
Funding There is no funding.

\section{Declarations}

Conflict of interest None of the authors have any conflict of interest.

\section{References}

1. Gracia-Ramos AE, Saavedra-Salinas MÁ (2021) Can the SARS$\mathrm{CoV}-2$ infection trigger systemic lupus erythematosus? A casebased review. RheumatolInt. https://doi.org/10.1007/s00296-02104794-7

2. Saadeh D, Kurban M, Abbas O (2016) Update on the role of plasmacytoid dendritic cells in inflammatory/autoimmune skin diseases. ExpDermatol 25(6):415-421

3. Saadeh D, Kurban M, Abbas O (2016) Plasmacytoid dendritic cell role in cutaneous malignancies. J DermatolSci 83(1):3-9
4. Sleiman R, Kurban M, Abbas O (2015) Evaluation of the diagnostic value of plasmacytoid dendritic cells in differentiating the lymphocytic cicatricial alopecias. Dermatology 231(2):158-163

5. Onodi F, Bonnet-Madin L, Meertens L et al (2021) SARS-CoV-2 induces human plasmacytoid pre-dendritic cell diversification via UNC93B and IRAK4. J Exp Med 218:e20201387

6. Battesti G, El Khalifa J, Abdelhedi N et al (2020) New insights in COVID-19-associated chilblains: a comparative study with chilblain lupus erythematosus. J Am AcadDermatol 83(4):1219-1222

7. Kim J-M, Park S-H, Kim H-Y, Kwok S-K (2015) A plasmacytoid dendritic cells-type I interferon axis is critically implicated in the pathogenesis of systemic lupus erythematosus. Int J MolSci 16(6):14158-14170

8. Tanboon J, Nishino I (2021) COVID-19-associated myositis may be dermatomyositis. Muscle Nerve 63(1):E9-E10

Publisher's Note Springer Nature remains neutral with regard to jurisdictional claims in published maps and institutional affiliations. 